UT-11-38

TU-894

IPMU-11-0188

\title{
Exploring Supersymmetric Model with Very Light Gravitino at the LHC
}

\author{
Masaki Asano ${ }^{(a, b)}$, Takumi Ito ${ }^{(b, c)}$, Shigeki Matsumoto ${ }^{(d)}$ \\ and Takeo Moroi ${ }^{(b, d)}$
}

(a) II. Institute for Theoretical Physics, University of Hamburg, Luruper Chausse 149, DE-22761 Hamburg, Germany

${ }^{(b)}$ Department of Physics, University of Tokyo, Tokyo 113-0033, Japan

(c) Department of Physics, Tohoku University, Sendai 980-8578, Japan

(d) IPMU, TODIAS, University of Tokyo, Kashiwa, 277-8583, Japan

\begin{abstract}
The low-scale gauge mediation scenario of supersymmetry breaking predicts very light gravitino, which makes the next lightest supersymmetric particle (NLSP) quasi stable. We study the LHC phenomenology of the case that the NLSP is the stau. When the mass of the gravitino is of the order of $10 \mathrm{eV}$, the decay length of stau is about $0.1-1 \mathrm{~mm}$, so that it decays before reaching the first layer of the inner silicon detector. We show, however, that, with utilizing the impact parameter of $\tau$-jets from the stau decay, it is possible to determine the mass spectrum of sparticles precisely. It is also possible to estimate the lifetime of the stau by observing distribution of the impact parameter.
\end{abstract}




\section{Introduction}

Large Hadron Collider experiment (LHC) is now operating and reports many important results on new physics beyond the standard model (SM). Although positive signals have not been reported so far, those are expected to be found in near future, because the hierarchy problem of the SM strongly suggests the existence of new physics at the TeV scale or below. On the other hand, many new physics models have been theoretically proposed. Among those, the supersymmetric model is very attractive because it guarantees the stability of the Higgs mass to its radiative corrections and gives a clue to solve the hierarchy problem. In addition, the supersymmetry (SUSY) plays a crucial role to realize the grand unification of known gauge interactions of the SM at a certain high energy scale.

Details of supersymmetric model, such as the mass spectrum of sparticles, depend highly on how SUSY is broken. So far, a variety of SUSY breaking mechanisms has been proposed [1]. Among those, the gauge mediation scenario [2] attracts an attention, because it gives a solution to dangerous SUSY flavor problems. In this scenario, the breaking occurs at lower energy scale than those of other SUSY breaking scenarios, so that the superpartner of graviton, the gravitino, is likely to be the lightest supersymmetric particle (LSP). The gravitino mass is predicted to be in the range between $\mathcal{O}(10) \mathrm{eV}$ and $\mathcal{O}(1) \mathrm{GeV}$. In this article, we focus on the low-scale gauge mediation model providing a gravitino with $\mathcal{O}(10) \mathrm{eV}$ mass. Such a scenario is well motivated because it is completely free from severe cosmological constraints [3] such as Big-Bang Nucleosynthesis [4] and large scale structure formation of our universe [5].

Collider signals of the low-scale gauge mediation scenario depend on what the next lightest superparticle (NLSP) is, which decays only into gravitino and its superpartner. Though there are many candidates for NLSP, we focus on the stau NLSP in this article, which is predicted in wide parameter region of the scenario. When the gravitino mass is of $\mathcal{O}(10) \mathrm{eV}$, the stau NLSP decays into a $\tau$-lepton and a gravitino with the lifetime of $10^{-15}-10^{-11}$ sec. The decay length (the lifetime times the speed of light) of the stau NLSP is therefore much shorter than the typical size of collider detectors, and the traditional supersymmetric signal, namely, multi-jets associated with missing energy and $\tau$-leptons, is expected at the LHC experiment. Such a signal is, however, generally predicted in various SUSY breaking scenarios. 
We show in this article that, even if the NLSP decays before reaching inner trackers of collider detectors, we can use the impact-parameter information about the decay products of the NLSP to study various properties of superparticles. In particular, the impact parameter is available for charged tracks caused by decay products of $\tau$-lepton at the stau NLSP decay. If the decay product of stau is found to have large impact parameter, it strongly suggests that the underlying SUSY breaking scenario is low-energy gauge mediation. Furthermore, the impact parameter is also utilized to precisely measure the spectrum of sparticles such as squark, neutralino, and stau masses. This is because two tau leptons produced by the cascade decay of a squark can be distinguished with each other by using the impact parameter. In addition, we may be able to determine the lifetime of the NLSP (i.e., stau in the present study) using the impact parameter distribution. When mass and lifetime of the stau NLSP are measured, it is possible to determine the gravitino mass assuming that the stau decays into gravitino and tau. The scale of SUSY breaking in the low-energy gauge mediation scenario is, therefore, obtained. It has been already shown that such studies can be easily performed once the $e^{+} e^{-}$linear collider becomes available [6]. Here, we consider the case of the LHC. We will see that the measurement of the mass spectrum as well as the determination of the lifetime of the NLSP can be performed at the LHC with the help of impact parameter information.

This article is organized as follows. In the next section, we consider some properties of the stau NLSP in the low-energy gauge mediation scenario and discuss how the impact parameter from the NLSP decay is utilized in determinations of sparticle masses and NLSP lifetime. Our simulation framework is summarized in section 3, in which a representative point and several strategies to reduce backgrounds are shown. In section 4, simulation results for the measurements of sparticle masses and lifetime of the stau NLSP are discussed. Section 5 is devoted to summary of our studies.

\section{Utilizing impact parameter}

In this section, we discuss how the impact parameter is utilized in order to determine the mass spectrum of sparticles and the lifetime of stau NLSP. We first briefly review some properties of the stau NLSP and define the impact parameter. Then, we discuss basic strategies for the measurement of the mass spectrum and the lifetime of NLSP 
with the use of the impact parameter.

\subsection{Stau NLSP and impact parameter}

Since the LHC is a hadron collider, colored sparticles such as squarks and gluino are expected to be produced at first, which decay into the stau NLSP, the super partner of $\tau$-lepton, through several cascade channels. The stau NLSP then decays into a $\tau$-lepton and a gravitino with the following lifetime,

$$
\tau_{\tilde{\tau}}=48 \pi M_{\mathrm{pl}}^{2}\left(\frac{m_{3 / 2}^{2}}{m_{\tilde{\tau}}^{5}}\right) \simeq 5.9 \times 10^{-12}[\mathrm{sec}] \times\left(\frac{m_{3 / 2}}{10 \mathrm{eV}}\right)^{2}\left(\frac{100 \mathrm{GeV}}{m_{\tilde{\tau}}}\right)^{5}
$$

where $M_{\mathrm{pl}} \simeq 2.4 \times 10^{18} \mathrm{GeV}, m_{\tilde{\tau}}$, and $m_{3 / 2}$ are reduced Planck mass, stau mass, and gravitino mass, respectively. It turns out from above formula that the decay length (the lifetime $\times$ the speed of light) of the stau NLSP is estimated to be $\sim \mathcal{O}(100) \mu \mathrm{m}$ when gravitino and stau masses are $\sim 10 \mathrm{eV}$ and $\sim 100 \mathrm{GeV}$, respectively. On the other hand, the decay length of $\tau$-lepton which is one of main backgrounds against the stau signal, is $87 \mu \mathrm{m}$, so that the decay of stau NLSP into very light gravitino can be, in principal, detected if we can reduce SM backgrounds efficiently.

Since the decay length of the stau NLSP is, at most, $\mathcal{O}(1) \mathrm{mm}$ in the parameter region of our interest, the stau NLSP decays before reaching the first pixel detector, which is located at $5 \mathrm{~cm}(4 \mathrm{~cm})$ away from the beam line in the ATLAS detector [7] (CMS detector [8]). The lifetime of the stau NLSP is, as a result, difficult to be measured using methods usually applied to detect long-lived particles, such as methods by observing charged tracks $[9,10,11,12]$. On the other hand, the lifetime of the stau NLSP may still be possible to be determined using the distribution of the impact parameter, which is obtained by $\tau$-jets from the stau NLSP decay. The impact parameter is defined as the shortest distance to the track from the interaction point. The positional resolution of the ATLAS detector along the longitudinal direction is $\Delta_{L} \sim 100 \mu \mathrm{m}$, which is not good compared to that along the transverse direction, $\Delta_{T} \sim 10 \mu \mathrm{m} .1$ Thus, we use the transverse impact parameter which is defined by

$$
d_{I} \equiv\left|\mathbf{x}_{T}^{I}-\frac{\mathbf{x}_{T}^{I} \cdot \mathbf{P}_{T}^{I}}{\left|\mathbf{P}_{T}^{I}\right|^{2}} \mathbf{P}_{T}^{I}\right|
$$

\footnotetext{
${ }^{1}$ Details of those performances are found in the section "Tracking" in Ref. [7].
} 


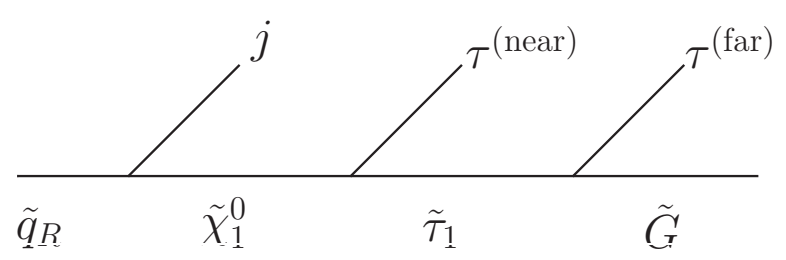

Figure 1: Typical decay chain in the low-scale gauge mediation scenario.

where $\mathbf{x}_{T}^{I}$ and $\mathbf{P}_{T}^{I}$ are transverse decay point of the $I$-th $\tau$-lepton and transverse momentum of the tau-jets from the $I$-th $\tau$-lepton decay, respectively. Note that the summation over the index $I$ should not be taken here. The above formula is used in our simulation studies, which will be presented in following sections. We expect that, at the LHC experiment, the distribution of the impact parameter $d_{I}$ is obtained by measuring the shortest distance (projected onto the transverse-plane) to the $\tau$-jet track from the interaction point.

\subsection{Impact parameter for mass measurements}

We next consider how the impact parameter is utilized in mass measurements of sparticles. At the LHC, colored sparticles such as gluino and squarks are expected to be produced copiously, and non-colored sparticles are then produced through cascade decays of the colored ones. The chain of the cascade decay is, for example, composed of following processes; First colored sparticle decays into a neutralino/chargino by emitting a quark which is observed as a jet. Next a neutralino/chargino decays into a slepton by emitting a lepton. Finally, a slepton decays into a LSP by again emitting a lepton. It is needless to say that the LSP passes through the detector without giving any signatures, which is, instead, observed as a missing energy. This chain (called "golden mode") is frequently used to measure the mass spectrum of sparticles in various supersymmetric scenarios by using several kinematical endpoints [14, 15].

In the case of the low-scale gauge mediation scenario, we have a similar decay chain. One of the examples is shown in Fig. 1, where $\tilde{q}_{R}, \tilde{\chi}_{1}^{0}, \tilde{\tau}_{1}$, and $\tilde{G}$ are righthanded squark, lightest neutralino, lightest stau, and gravitino, respectively. The character $j$ denotes a jet which originates in a quark from the $\tilde{q}_{R}$ decay 2 Following

\footnotetext{
${ }^{2}$ In the gluino production event, the gluino decays into a squark by also emitting a jet. Because the mass difference between gluino and squark is much smaller than that between squark and
} 
the terminology used in studies of the golden mode, we call $\tau$ from the $\tilde{\chi}_{1}^{0}$-decay $\tau^{\text {(near) }}$ and that from the $\tilde{\tau}_{1}$-decay $\tau^{\text {(far) }}$. Importantly, the impact parameter of $\tau^{\text {(far) }}$ is expected to be larger than that of $\tau^{\text {(near) }}$, which is of great help for the event reconstruction.

In our analysis, we focus on the signal from the decay of a squark shown in Fig. 1. Kinematics of its decay chain is, as a result, governed by following four sparticle masses; the masses of squark $\left(m_{\tilde{q}}\right)$, lightest neutralino $\left(m_{\tilde{\chi}_{1}^{0}}\right)$, lightest stau $\left(m_{\tilde{\tau}_{1}}\right)$, and gravitino $\left(m_{3 / 2}\right)$. Since the gravitino mass is of the order of $10 \mathrm{eV}$, only the upper bound on the mass is expected to be obtained. On the other hand, the existence of large impact parameters in signal events strongly suggests the (low-scale) gauge mediation scenario. We therefore perform our analysis with simply postulating that the gravitino mass is much smaller than those of other sparticles, namely, with treating the gravitino as a massless particle. Three independent kinematical endpoints are then enough to determine the mass spectrum of sparticles. With the help of the impact parameter, many kinematical variables are now available. Among those, we use the invariant mass between two $\tau$-leptons $\left(M_{\left.\tau^{\text {(near })} \tau_{\text {(far) }}\right)}\right.$, that between jet and near $\tau$-lepton $\left(M_{j \tau^{(\text {near }}}\right)$, and the $M_{T 2}$ variable from leading two jets $\left(M_{T 2, j j}\right)$.

The upper limit on the invariant mass $M_{\tau^{(\text {near })} \tau^{\text {(far) }}}$ is given by

$$
M_{\tau^{(\text {near })} \tau^{(\text {far })}}^{\max }=m_{\tilde{\chi}_{1}^{0}} \sqrt{1-m_{\tilde{\tau}_{1}}^{2} / m_{\tilde{\chi}_{1}^{0}}^{2}}
$$

where the mass of $\tau$-lepton is set to be zero in above formula. In addition, the upper limit on the distribution of the invariant mass $M_{j \tau}$ (near) is given by the following formula,

$$
M_{j \tau^{(\text {near })}}^{\max }=m_{\tilde{q}} \sqrt{\left(1-m_{\tilde{\chi}_{1}^{0}}^{2} / m_{\tilde{q}}^{2}\right)\left(1-m_{\tilde{\tau}_{1}}^{2} / m_{\tilde{\chi}_{1}^{0}}^{2}\right)}
$$

The last kinematical variable used in our analysis is the $M_{T 2}$ variable [16] constructed from highest two jets; denoting the momenta of highest two jets as $\mathbf{p}$ and $\mathbf{p}^{\prime}$, we define

$$
M_{T 2, j j}\left(m_{\mathrm{miss}}\right)=\min _{\mathbf{k}_{T}+\mathbf{k}_{T}^{\prime}=\mathbf{p}_{T}^{\text {eff }}}\left[\max \left\{M_{T}\left(\mathbf{p}_{T}, \mathbf{k}_{T}\right), M_{T}\left(\mathbf{p}_{T}^{\prime}, \mathbf{k}_{T}^{\prime}\right)\right\}\right]
$$

where $M_{T}$ is the transverse mass and $m_{\text {miss }}$ is the "test mass." Here, because we construct the $M_{T 2}$ variable only from highest two jets, $\mathbf{P}_{T}^{\text {eff }}$ should be understood as neutralino in the parameter region of our interest, we can discriminate between a (soft) jet from the gluino decay and a (hard) jet from the squark decay. 
the vector sum of transverse momenta of all the activities other than highest two jets and missing momentum $\boldsymbol{p}_{T}$ : $\mathbf{P}_{T}^{\text {eff }}=\boldsymbol{p}_{T}+\sum_{i} \mathbf{p}_{\tau-\text { jet } i}+\sum_{i} \mathbf{p}_{\tau-j e t i}^{\prime}$. The upper limit of this variable is then given by

$$
M_{T 2, j j}^{\max }\left(m_{\mathrm{miss}}\right)=\frac{m_{\tilde{q}}^{2}-m_{\tilde{\chi}_{1}^{0}}^{2}}{2 m_{\tilde{q}}}+\sqrt{\left(\frac{m_{\tilde{q}}^{2}-m_{\tilde{\chi}_{1}^{0}}^{2}}{2 m_{\tilde{q}}}\right)^{2}+m_{\mathrm{miss}}^{2}} .
$$

Using three kinematical endpoints given in eqs.(3), (41) and (66), we fit the sparticle masses $m_{\tilde{q}}, m_{\tilde{\chi}_{1}^{0}}$, and $m_{\tilde{\tau}_{1}}$. We will see that, though the cascade chain always involves $\tau \mathrm{s}$ as lepton emissions, the spectrum can be determined accurately because of information about the impact parameter.

\subsection{Impact parameter for stau lifetime measurement}

Information about the lifetime of the stau NLSP is imprinted in the distribution of the impact parameter. The impact parameter, however, depends not only on the lifetime of the stau NLSP but also on its mass and velocity. With the use of the strategy discussed in previous subsection, the stau mass is measured precisely. On the other hand, since the gravitino produced from the stau decay cannot be detected, the velocity cannot be determined on event-by-event basis, which makes it difficult to determine the lifetime using the impact parameter distribution.

Once the mass spectrum of the superparticles are known, however, we expect to acquire information about the velocity distribution of the $\tilde{\tau}_{1}$ in supersymmetric events. In the present case (where the mass spectrum of a simple gauge mediation model is assumed), we may understand that the underlying scenario is indeed the low-scale gauge mediation from the experimentally measured mass spectrum as well

as the confirmation of the existence of long-lived stau. Even if we cannot specify the complete structure of the underlying model, we may still be able to measure the masses of superparticles which are most important for the determination of the velocity distribution of $\tilde{\tau}_{1}$ (i.e., the masses of $\tilde{q}, \tilde{\chi}_{1}^{0}$, and $\tilde{\tau}_{1}$ ), as we have discussed in the previous section. Then, once those information becomes available, one will be able to obtain the velocity distribution with, for example, Monte Carlo simulation.

In our analysis, we assume that the velocity distribution of $\tilde{\tau}_{1}$ can be understood once the superparticles are discovered. The detailed study of the methods of determining the velocity distribution is beyond the scope of this paper, so we simply 
assume that the averaged velocity of the produced $\tilde{\tau}_{1}$ can be obtained with some accuracy and determine the lifetime using the $\tilde{\tau}_{1}$. Although a better determination of the lifetime of $\tilde{\tau}_{1}$ may be possible if we can somehow obtain and use the information about the velocity distribution of $\tilde{\tau}_{1}$, we can still have a relatively good determination of the lifetime using the averaged velocity as we will describe. The procedure to measure the lifetime of the stau NLSP is therefore the following.

(i) We first assume that the averaged velocity of the stau NLSP, denoted as $\bar{\beta}_{\tilde{\tau}_{1}}$, is somehow understood. Then, we generate $\tilde{\tau}_{1}$ with the fixed velocity $\bar{\beta}_{\tilde{\tau}_{1}}$ and make signal templates of the distribution of the transverse impact parameter $d_{I}$. The template is prepared for wide range of stau lifetime. The production angle of the stau NLSP is assumed to be isotropic in generating the events for the template.

(ii) Distribution of the impact parameter expected at the LHC experiment is obtained by using Monte Carlo simulation.

(iii) Comparing the templates obtained in the (i) with the actual distribution obtained in (ii), we study how well we can constrain the lifetime of $\tilde{\tau}_{1}$ by $\chi^{2-}$ analysis. By varying the value of $\bar{\beta}_{\tilde{\tau}_{1}}$ used in making the templates, we also discuss the uncertainty related to the determination of the velocity distribution.

Here, we have a few comments on the above method. First comment is on the effect of gluino production. The mass difference between gluino and squark is much smaller than that between squark and neutralino in the parameter region of our interest. In addition, it will be possible to select signal events with the desirable squark decay chain $\left(\tilde{q} \rightarrow \tilde{\chi}_{1}^{0} \rightarrow \tilde{\tau}_{1} \rightarrow \tilde{G}\right)$ by applying appropriate kinematical cuts. Thus, the averaged value of the boost factor is expected to depend weakly on the gluino mass. We have checked this statement quantitatively by simulating signal events with several choices of the gluino mass. Second comment is on how the transverse impact parameter $d_{I}$ depends on the production angle of the stau NLSP. One might worry if we may compare the actual impact parameter distribution with theoretical templates obtained by postulating isotropic distribution of the production angle. We have checked that this potential problem can be solved by only using $\tau$-jets with small pseudo-rapidity. 


\section{Simulation framework}

Before showing our results, we summarize the framework of our simulation study. We first mention a representative point and simulation tools used in the study. Next we discuss the strategy to suppress combinatorial backgrounds of signal events caused by the existence of two decay chains. We finally consider the SM backgrounds and discuss kinematical cuts used to reduce those backgrounds.

\subsection{Representative point \& simulation tools}

The representative point used in our simulation study has been chosen by adopting the minimal model of the gauge mediation symmetry breaking [2] with the following underlying parameters; the SUSY breaking scale $(\Lambda=30 \mathrm{TeV})$, the messenger mass scale $\left(M_{\text {mess }}=300 \mathrm{TeV}\right)$, the number of SU(5) messenger fields $\left(N_{\mathbf{5}}=5\right)$, and the ratio of vacuum expectation values of two Higgs fields $(\tan \beta=15)$. The ISAJET package [17] is used in order to calculate the spectrum and branching fractions of sparticles. Resultant masses and branching fractions of sparticles relevant to the study are summarized in Table 1 for the case of the gravitino mass of $9.7 \mathrm{eV}$ which corresponds to the decay length of the stau of $500 \mu \mathrm{m}$. It can be seen that the model is consistent with current LHC data [13].

In our analysis, we consider the LHC experiment with the center of mass energy of $\sqrt{s}=14 \mathrm{TeV}$. Then, the signal cross section, which is the sum of the cross sections for gluino and squark productions, is estimated to be

$$
\sigma_{\tilde{g} \tilde{g}}=0.129 \mathrm{pb}, \quad \sigma_{\tilde{q} \tilde{g}}=0.922 \mathrm{pb}, \quad \sigma_{\tilde{q} \tilde{q}}=0.879 \mathrm{pb}
$$

We focus on the decay chain involving a right-handed squark, as shown in Fig. 1. As a result, a typical signal event consists of two energetic jets, four $\tau$-leptons ( $\tau$-jets or leptons), and a missing energy in the transverse direction.

For parton-level event generation and hadronization, we employ the HERWIG code [18, 19]. Generated events are passed through the PGS code [20] for simulating detector effects. Fake $\tau$-jets from QCD processes and heavy meson decays are involved in the study. For tau-jets, we smear the transverse vertex position of the parton using Gaussian distribution with the error $\Delta d_{I}=10 \mu \mathrm{m}$. 


\begin{tabular}{c|cll} 
& Mass $(\mathrm{GeV})$ & \multicolumn{1}{|c}{ Branching fractions } \\
\hline$\tilde{g}$ & 1096.6 & $\operatorname{Br}(\tilde{g} \rightarrow \tilde{q} q)=0.89$. \\
$\tilde{u}_{L}$ & 951.1 & $\operatorname{Br}\left(\tilde{u}_{L} \rightarrow \tilde{\chi}_{2}^{ \pm} d\right)=0.34, \quad \operatorname{Br}\left(\tilde{u}_{L} \rightarrow \tilde{\chi}_{1}^{ \pm} d\right)=0.32$, \\
& & $\operatorname{Br}\left(\tilde{u}_{L} \rightarrow \tilde{\chi}_{4}^{0} u\right)=0.18, \quad \operatorname{Br}\left(\tilde{u}_{L} \rightarrow \tilde{\chi}_{2}^{0} u\right)=0.15$. \\
$\tilde{u}_{R}$ & 922.0 & $\operatorname{Br}\left(\tilde{u}_{R} \rightarrow \tilde{\chi}_{1}^{0} u\right)=0.96$. \\
$\tilde{\chi}_{1}^{0}$ & 197.3 & $\operatorname{Br}\left(\tilde{\chi}_{1}^{0} \rightarrow \tilde{\tau}_{1}^{ \pm} \tau^{\mp}\right)=0.35, \quad \operatorname{Br}\left(\tilde{\chi}_{1}^{0} \rightarrow \tilde{e}_{R}^{ \pm} e^{\mp}\right)=0.32$, \\
& & $\operatorname{Br}\left(\tilde{\chi}_{1}^{0} \rightarrow \tilde{\mu}_{R}^{ \pm} \mu^{\mp}\right)=0.32$. \\
$\tilde{e}_{R}$ & 130.0 & $\operatorname{Br}\left(\tilde{e}_{R} \rightarrow \tilde{\tau}_{1}^{ \pm} \tau^{\mp} e\right) \simeq 1.00$. \\
$\tilde{\tau}_{1}$ & 126.2 & $\operatorname{Br}\left(\tilde{\tau}_{1} \rightarrow \tau \tilde{G}\right)=1.00$. \\
\hline
\end{tabular}

Table 1: Masses and branching fractions of sparticle in our representative point.

\subsection{Charge subtraction method}

Background reduction is the most important task in our analysis to determine the mass spectrum of sparticles, because all kinematical endpoints do not have sharp edge structures due to the energy leakage by $\nu_{\tau}$ emissions from $\tau$-decays. Expected backgrounds in those measurements are as follows: (i) A number of fake $\tau$-jets are expected at the hadron collider. (ii) A signal event results in multiple $\tau$-leptons, and hence there are combinatorial backgrounds in the analysis involving $\tau$-jets.

In order to reduce these backgrounds, we adopt the method of the charge subtraction. We expect four $\tau$-leptons (two $\tau^{+}$and two $\tau^{-}$) in one event. We therefore have three ways to pair the $\tau$ leptons, $\left(\tau^{ \pm}, \tau^{\mp}\right)_{1},\left(\tau^{ \pm}, \tau^{\mp}\right)_{2}$, and $\left(\tau^{ \pm}, \tau^{ \pm}\right)$. In each event (involving four $\tau \mathrm{S}$ ), we take the data using the method $\left(\tau^{ \pm}, \tau^{\mp}\right)_{1}+\left(\tau^{ \pm}, \tau^{\mp}\right)_{2}-\left(\tau^{ \pm}, \tau^{ \pm}\right)$, then the wrong opposite-sign pairing is expected be canceled by the subtraction of the same-sign pairing. This method works very well when $\tau$-leptons are produced in the process $\tilde{\chi}^{0} \rightarrow \tilde{\tau} \rightarrow \tilde{G}$; this is due to the fact that $\tilde{\chi}^{0}$ decays into $\tilde{\tau}^{+} \tau^{-}$and $\tilde{\tau}^{-} \tau^{+}$ with equal probability.

In addition, this method can be applied to the determination of the $M_{j \tau}$ (near)endpoint. We simply collect $(j, \tau, \tau)$ events using the charge subtraction. In each paring, $\tau^{\text {(near) }}$ is identified as the $\tau$ lepton which has a shorter impact parameter. It should be also noted that the charge subtraction method can reduce backgrounds from fake $\tau$-jets from QCD processes, because the QCD fake events are charge-blind in a good approximation at high energy processes. 


\begin{tabular}{l|rr} 
Selection cut & SUSY & $t \bar{t}$ \\
\hline (0) Generated events & 273,600 & $49,610,000$ \\
$(1) p_{T}>150 \mathrm{GeV}$ & 190,504 & $1,644,160$ \\
$(2) \#$ of leptons $\geq 4$ & 70,641 & 55,537 \\
$(3) \#$ of b-jets $=0$ & 56,228 & 41,673 \\
$(4) j_{1}$ with $p_{T}>200 \mathrm{GeV}$ & 49,819 & 5,915 \\
$(5) j_{2}$ with $p_{T}>100 \mathrm{GeV}$ & 41,007 & 1,984 \\
\hline
\end{tabular}

Table 2: Numbers of signal (SUSY) and background $(t \bar{t})$ events after applying kinematical cuts with $\mathcal{L}=100 \mathrm{fb}^{-1}$. All SUSY processes are included in our event generation.

\subsection{Kinematical cuts to reduce $t \bar{t}$ backgrounds}

The most serious SM background for our study is the $t \bar{t}$ production. Thus, we concentrate on this background. In order to reduce this background, we impose following kinematical cuts:

- Large missing transverse energy, $\not \not_{T}>150 \mathrm{GeV}$.

- At least, four leptons, $e, \mu$ with $p_{T}>20 \mathrm{GeV}$ or $\tau$-jet with $p_{T}>25 \mathrm{GeV}$.

- Two hard jets, $j_{1}\left(j_{2}\right)$ with $p_{T}>200(100) \mathrm{GeV}$ (and no b-jets).

Here, we use the label, $i=1$ or 2 , for a jet $\left(j_{i}\right)$ in decreasing order of $p_{T}$. We also take account of both electrons and muons, because $\tau$-lepton often decays leptonically and selectron and smuon decay into gravitino by emitting electron and muon directly. The requirement for jets to have $p_{T}>200(100) \mathrm{GeV}$ is very important to reduce the $t \bar{t}$ background, because jets with $p_{T}>m_{W}\left(m_{W}\right.$ is the mass of weak gauge boson) from top quark decays are rather rare. In Table 2, we summarize the cut flow in our simulation study with assuming that the integrated luminosity is $100 \mathrm{fb}^{-1}$, where we take the gravitino mass of $9.7 \mathrm{eV}$ again.

It is possible to apply tighter kinematical cuts for further reductions of the backgrounds. For instance, the requirement $p_{T}>200 \mathrm{GeV}, p_{T}\left(j_{1}\right)>250 \mathrm{GeV}$ and $p_{T}\left(j_{2}\right)>$ $150 \mathrm{GeV}$ in addition to the basic kinematical cuts shown in Table 2 will reduce $80 \%$ of the $t \bar{t}$ background (we have, as a result, $\sim 400 t \bar{t}$ events), while this also reduces $30 \%$ of SUSY signals (we have, as a result, $\sim 28000$ SUSY events). 


\section{Simulation results}

We are now in position to present several results of our simulation study, which are obtained based on arguments in previous sections. We first show the results for measurements of sparticle masses, and discuss how accurately these masses can be determined at the LHC. We next show that the lifetime of the stau may be determined by using the distribution of the transverse impact parameter of $\tau$-jets from the stau NLSP decay. We estimate how accurately the lifetime can be determined.

\subsection{Sparticle masses}

The strategy to determine the mass spectrum is the use of kinematical endpoints of several variables. We study how the endpoints behaves using generated events which pass through the basic cuts discussed in section 3.3. For the simulation study of sparticle mass measurement, the decay length of the stau NLSP is set to be $500 \mu \mathrm{m}$ (corresponding to the gravitino mass of $9.7 \mathrm{eV}$ ).

\subsubsection{Endpoint on $M_{\tau \tau}$}

The first kinematical variable used in the analysis for the mass spectrum is the invariant mass of two $\tau$-leptons in the decay chain of a squark. In Fig. 2 (upper panel), the distribution of the invariant mass $\left(M_{\tau \tau}\right)$ after applying the charge subtraction method is shown. We can see a clear edge at $M_{\tau \tau} \simeq 150 \mathrm{GeV}$. In order to extract the location of the endpoint, we use the following fitting function,

$$
f\left(M_{\tau \tau}\right)=\left\{\begin{array}{ll}
A\left(M_{\tau \tau}-M_{\tau \tau}^{\mathrm{fit}}\right)+C & : M_{\tau \tau}<M_{\tau \tau}^{\mathrm{fit}} \\
B\left(M_{\tau \tau}-M_{\tau \tau}^{\mathrm{fit}}\right)+C & : M_{\tau \tau}>M_{\tau \tau}^{\mathrm{fit}}
\end{array},\right.
$$

where $A, B, M_{\tau \tau}^{\mathrm{fit}}$, and $C$ are parameters to fit the shape of the distribution around the endpoint. With the use of this bilinear function for the fitting, the location of the endpoint is determined to be $M_{\tau \tau}^{\text {fit }}=151.2 \pm 14.5 \mathrm{GeV}$. Notice that the underlying value (the input value on the simulation) is $151.6 \mathrm{GeV}$.

\subsubsection{Endpoint on $M_{q \tau^{(\text {near }}}$}

Second kinematical variable we use is the invariant mass between $\tau$-lepton and jet emitted by the decay of a squark. Using information about the impact parameter, it 

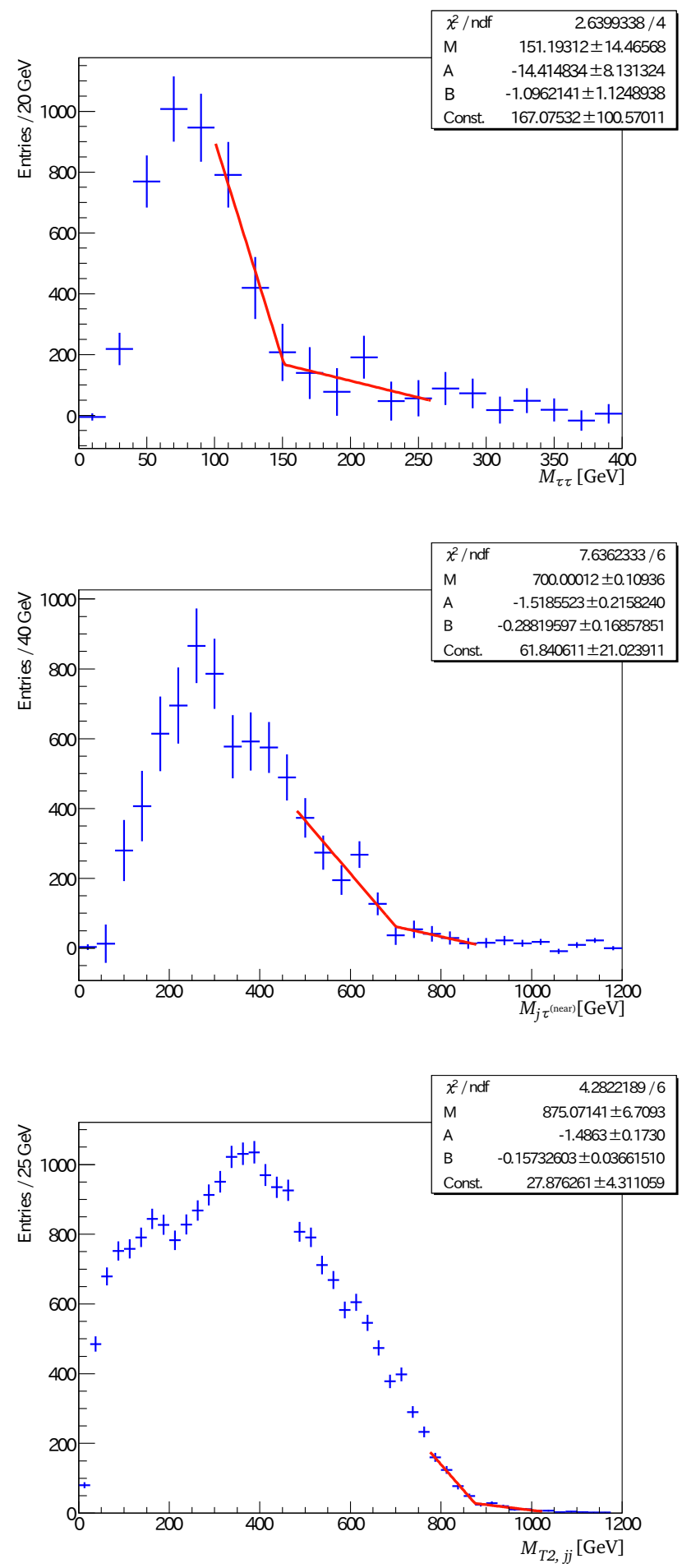

Figure 2: (Upper panel) Distribution of the invariant mass between two tau-jets, $M_{\tau \tau}$. (Middle panel) Distribution of the invariant mass between hard jet and near $\tau$-jet, $M_{j \tau^{\text {(near) }}}$. (Lower panel) Distribution of the $M_{T 2, j j}$ variable defined in Eq. (6) with $m_{\text {miss }}$ being zero. 
is possible to distinguish near and far $\tau$-leptons with high efficiency. For each $(\tau, \tau)$ pair, we identify the $\tau$-jet whose track has a larger impact parameter than the other as the far tau-jet, while the $\tau$-jet with a smaller impact parameter is regarded as the near $\tau$-jet. After this identification, both combinations of $\left(j_{1}, \tau^{(\text {near })}\right)$ and $\left(j_{2}, \tau^{(\text {near })}\right)$ are used to calculate $M_{q \tau^{(n e a r)}}$. In the analysis, we also require that the pair of two tau-jets should satisfy $M_{\tau \tau}<M_{\tau \tau}^{\text {fit }}=155.72 \mathrm{GeV}$ in order to reduce fake-QCD and combinatorial backgrounds.

The distribution of the invariant mass $\left(M_{q \tau^{(\text {near }}}\right)$ after applying the charge subtraction method is shown in Fig. 2 (middle panel). The endpoint is, again, fitted by using the bilinear function given in Eq. (8) . It then turns out that the location of the endpoint on $M_{q \tau^{(\text {near })}}$ is $M_{q \tau^{\text {(near }}}^{\text {fit }}=700.0 \pm 0.1 \mathrm{GeV}$. Notice that the underlying value is now $692.3 \mathrm{GeV}$.

\subsubsection{Endpoint on $M_{T 2, j j}$}

The last kinematical variable is $M_{T 2, j j}$ defined by two hard jets as visible particles, as mentioned in section 2.2. In our analysis, we take the test mass $\left(m_{\text {miss }}\right)$ in Eq. (6) to be zero, so that the endpoint of this kinematical variable gives $M_{T 2, j j}^{\max }(0)=\left(m_{\tilde{q}}^{2}-\right.$ $\left.m_{\tilde{\chi}_{1}^{0}}^{2}\right) / m_{\tilde{q}}$. The distribution of $M_{T 2, j j}(0)$ is shown in Fig. 2 (lower panel). It can be seen that a very clear endpoint exists at $M_{T 2, j j}(0) \simeq 870 \mathrm{GeV}$. As in the cases of previous kinematical variables, we fit the shape of the distribution around the endpoint by the bilinear function. The endpoint of the distribution is then obtained as $M_{T 2, j j}^{\text {fit }}(0)=875.1 \pm 6.7 \mathrm{GeV}$ (the underlying value is $879.8 \mathrm{GeV}$ ).

\subsubsection{Mass determination}

When the gravitino mass is neglected, the masses of sparticles, $m_{\tilde{q}}, m_{\tilde{\chi}_{1}^{0}}$, and $m_{\tilde{\tau}_{1}}$, are determined by three kinematical endpoints of the variables, $M_{\tau \tau}^{\max }, M_{q \tau^{\text {(near) }}}^{\max }$, and $M_{T 2, j j}^{\max }(0)$. Using analytic expressions for the these endpoints shown in eqs.(3)), (41), and ([6), the masses of sparticles are determined by minimizing the following $\chi^{2}$ function,

$$
\chi_{\mathrm{m}}^{2}=\left[\frac{M_{\tau \tau}^{\mathrm{max}}-M_{\tau \tau}^{\mathrm{fit}}}{\Delta M_{\tau \tau}^{\mathrm{fit}}}\right]^{2}+\left[\frac{M_{q \tau^{\text {(near })}}^{\mathrm{max}}-M_{q \tau^{\text {(near })}}^{\mathrm{fit}}}{\Delta M_{q \tau^{\text {(near })}}^{\text {fit }}}\right]^{2}+\left[\frac{M_{T 2, j j}^{\max }(0)-M_{T 2, j j}^{\mathrm{fit}}(0)}{\Delta M_{T 2, j j}^{\mathrm{fit}}(0)}\right]^{2},
$$

where $M^{\text {fit }}$ denotes the center value of the measured endpoint, and $\Delta M^{\text {fit }}$ is its

(statistical) error. After minimizing $\chi_{\mathrm{m}}^{2}$ by varying the input values, $m_{\tilde{q}}, m_{\tilde{\chi}_{1}^{0}}$, and 


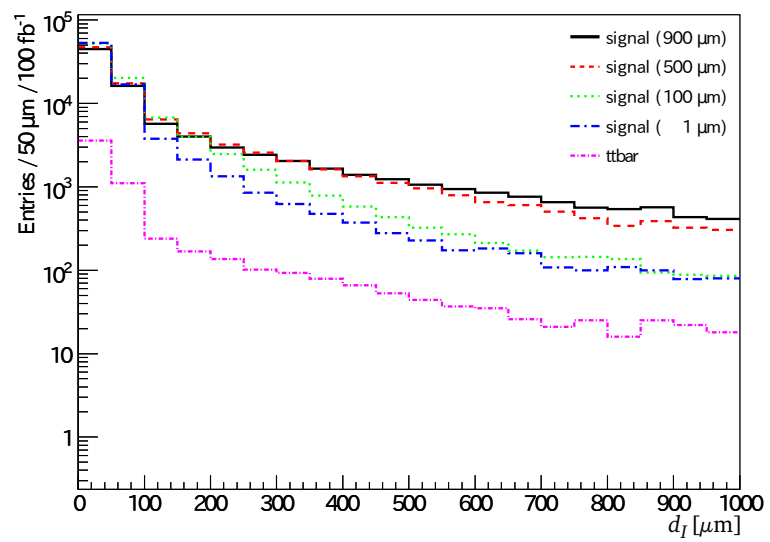

Figure 3: Distribution of the transverse impact parameter of $\tau$-jets after applying the basic kinematical cuts in previous section. We show distributions in the SUSY model with $c \tau_{\tilde{\tau}}=1,100,500$ and $900 \mu \mathrm{m}$, respectively, and also show the distribution in $t \bar{t}$ production.

$m_{\tilde{\tau}_{1}}$, we obtain following results; the right-handed squark mass is $m_{\tilde{q}}=915.9 \pm$ $6.4 \mathrm{GeV}$ (the true value is $922.0 \mathrm{GeV}$ ), the lightest neutralino mass is $m_{\tilde{\chi}_{1}^{0}}=193.4 \pm$ $19.5 \mathrm{GeV}$ (the true value is $197.3 \mathrm{GeV}$ ), and the lightest stau mass is $m_{\tilde{\tau}_{1}}=120.5 \pm$ $18.1 \mathrm{GeV}$ (the true value is $126.2 \mathrm{GeV}$ ). Here, the gravitino mass is taken to be zero (i.e., negligibly small).

\subsection{Lifetime of the stau NLSP}

The strategy to determine the lifetime of the stau NLSP is the use of the distribution of the transverse impact parameter. After showing the distribution for several input values of $c \tau_{\tilde{\tau}}$, we discuss how accurately the lifetime can be determined at the LHC.

\subsubsection{Distribution of the impact parameter}

Distribution of the transverse impact parameter $\left(d_{I}\right)$ obtained from hadronically decays of $\tau$-leptons is shown in Fig. 3 with the use of generated events which are passed through the kinematical cuts discussed in previous section. Four distributions are shown in this figure with choices of the decay length of the stau NLSP to be $c \tau_{\tilde{\tau}}=$ $1,100,500$, and $900 \mu \mathrm{m}$, respectively. It is clearly seen that the distribution depends on the decay length of the stau NLSP as expected. 
It is also seen that a number of events are found in inner bins with $d_{I} \ll c \tau_{\tilde{\tau}}$ and that a broad tail-structure exists in the region $d_{I} \gg c \tau_{\tilde{\tau}}$. In fact, in both regions, backgrounds are expected to contribute to the distribution. In small $d_{I}$ region, the distribution is dominated by background $\tau$-jets such as QCD-originated fake ones. Though most of those backgrounds do not have finite $d_{I}$ at the parton-level, the backgrounds acquire finite values of $d_{I}$ at the detector-level because of the limited resolution for the vertexing. On the other hand, in the region of $d_{I} \gg c \tau_{\tilde{\tau}}$, backgrounds come from decays of heavy hadrons such as $D$ or $B$ mesons. Those hadrons sometimes produce fake $\tau$-jets after flying a sizable distance. In order to eliminated these backgrounds efficiently, we vary and optimize the upper and lower endpoints

of the bins which are used for the $\chi^{2}$ analysis as we change $c \tau_{\tilde{\tau}}^{\text {(test) }}$ (where $c \tau_{\tilde{\tau}}^{\text {(test) }}$ is the test value of the decay length used to generate a template of $d_{I}$ distribution).

\subsubsection{Lifetime estimation}

According to the strategy to estimate the lifetime discussed in section 2.3, we now study how well we can constrain the lifetime of $\tilde{\tau}_{1}$. First, we use the template generated with the true value of the averaged velocity of the stau NLSP, which is $\bar{\beta}_{\tilde{\tau}}=0.88 c$ in our representative point. In order to see how the result depends on the underlying value of the lifetime of $\tilde{\tau}_{1}$, here we use several values of $c \tau_{\tilde{\tau}}$ in generating the impact-parameter distribution. In addition, in preparing the templates for $d_{I}$ distributions, TAUOLA library [21] is used to simulate the $\tau$ decay event, which enable us to deal with chirality and finite lifetime of $\tau$-leptons. The range of the test value is taken to be $10 \mu \mathrm{m}-1100 \mu \mathrm{m}$ every $10 \mu \mathrm{m}$.

With the use of the templates, we perform $\chi^{2}$-analysis to determine the lifetime. In our analysis, only $\tau$-jets satisfying $0.5 \times c \tau_{\tilde{\tau}}^{\text {(test) }}<d_{I}<2.0 \times c \tau_{\tilde{\tau}}^{\text {(test) }}$ with small pseudo-rapidity, $|\eta|<1.0$, are adopted. The size of bin used in each distribution is set to be $0.15 \times c \tau_{\tilde{\tau}}^{\text {(test) }}$ and, as a result, we have 10 bins in total. The $\chi^{2}$ variable to estimate the lifetime of the stau NLSP is therefore given by

$$
\chi^{2}\left(c \tau_{\tilde{\tau}}^{\text {(test) }}\right) \equiv \sum_{i=1}^{10}\left[\frac{N_{i}^{(\text {th })}\left(c \tau_{\tilde{\tau}}^{(\text {test })}\right)-N_{i}^{(\exp )}}{\Delta N_{i}}\right]^{2},
$$

where $N_{i}^{(\text {th) }}\left(c \tau_{\tilde{\tau}}^{\text {(test) }}\right)$ denotes the number of signals in the $i$-th bin obtained by the template for a given $c \tau_{\tilde{\tau}}^{\text {(test) }}$, while $N_{i}^{(\text {(exp) }}$ is the one obtained by using generated 


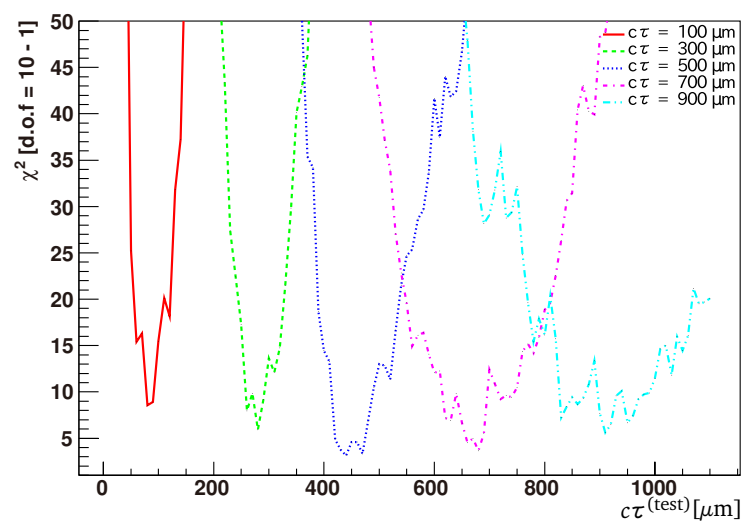

Figure 4: $\chi^{2}$-values as a function of the test lifetime (the test decay length) of the stau $\mathrm{NLSP}, c \tau_{\tilde{\tau}}^{\text {(test) }}$, for the underlying values of $c \tau_{\tilde{\tau}}=100$ (red, solid), 300 (green, dashed), 500 (blue, dotted), 700 (violet, dot-dashed), and 900 (cyan, dot-dot-dashed) $\mu \mathrm{m}$. Here, the averaged velocity is taken to be $\bar{\beta}_{\tilde{\tau}}=0.88$.

events. We only involves the statistical error as $\Delta N_{i} \equiv \sqrt{N_{i}^{(\exp )}}$ in the analysis. The degrees of the freedom in this $\chi^{2}$-test is therefore $(10-1)=9$, and the hypothesis is excluded at $95 \%$ C.L. when $\chi^{2}>16.92$.

Resultant $\chi^{2}$-values as a function of the test lifetime (the test decay length) of the stau NLSP, $c \tau_{\tilde{\tau}}^{\text {(test) }}$, for underlying values of $c \tau_{\tilde{\tau}}=100$ (red, solid), 300 (green, dashed), 500 (blue, dotted), 700 (violet, dot-dashed), and 900 (cyan, dot-dot-dashed) $\mu \mathrm{m}$ are shown in Fig. 4. From these results, the lifetime (the decay length) of the stau NLSP in each case is determined at $95 \%$ C.L. to be

$$
\begin{array}{rlrl}
50 \mu \mathrm{m} & \lesssim c \tau_{\tilde{\tau}} \lesssim 110 \mu \mathrm{m} & & \text { (underlying } \left.c \tau_{\tilde{\tau}}=100 \mu \mathrm{m}\right), \\
240 \mu \mathrm{m} & \lesssim c \tau_{\tilde{\tau}} \lesssim 330 \mu \mathrm{m} & & \text { (underlying } \left.c \tau_{\tilde{\tau}}=300 \mu \mathrm{m}\right), \\
410 \mu \mathrm{m} & \lesssim c \tau_{\tilde{\tau}} \lesssim 540 \mu \mathrm{m} & & \text { (underlying } \left.c \tau_{\tilde{\tau}}=500 \mu \mathrm{m}\right), \\
570 \mu \mathrm{m} & \lesssim c \tau_{\tilde{\tau}} \lesssim 800 \mu \mathrm{m} & \text { (underlying } \left.c \tau_{\tilde{\tau}}=700 \mu \mathrm{m}\right) \\
810 \mu \mathrm{m} & \lesssim c \tau_{\tilde{\tau}} \lesssim 1060 \mu \mathrm{m} & \text { (underlying } \left.c \tau_{\tilde{\tau}}=900 \mu \mathrm{m}\right)
\end{array}
$$

We can see that, if the correct value of $\bar{\beta}_{\tilde{\tau}}$ is used, the analysis based on the impact parameter distribution gives a good estimate of the lifetime (the decay length) of the stau NLSP with accuracy of about $30 \%$ when $c \tau_{\tilde{\tau}}>100 \mu \mathrm{m}$. If more precise information about the velocity distribution of $\tilde{\tau}_{1}$ is available, better estimate of the lifetime may be obtained. 
So far, we have neglected the uncertainty arising from the determination of the velocity distribution of $\tilde{\tau}_{1}$. As we have mentioned, the detailed study of the uncertainty in the velocity distribution is beyond the scope of this paper. In our analysis, however, we estimated the uncertainty of the $\bar{\beta}_{\tilde{\tau}}$ determination related to the errors in mass measurements and also to the production process in order to demonstrate that the $\bar{\beta}_{\tilde{\tau}}$ can be obtained with some accuracy.

First, in order to study the effects of the errors in the mass measurements, we generated the events using the different sparticle mass spectrum from our representative point. Here, we used the mass spectrum predicted from the simple gauge mediation model except for $\tilde{\tau}_{1}$ because we found that the error of $m_{\tilde{\tau}_{1}}$ is the largest among the reconstructed masses in the previous subsection. Then we generated the full SUSY events by varying $m_{\tilde{\tau}_{1}}$ by $\pm 20 \mathrm{GeV}$, and found that the value of $\bar{\beta}_{\tilde{\tau}}$ changes by $\sim 0.01$. In addition, the dominant SUSY process may not be well understood in the actual situation. If so, it may be reasonable to estimate $\bar{\beta}_{\tilde{\tau}}$ by assuming the process we use for our analysis, which is the process shown in Fig. 1, We generated events corresponding to such a process (using the correct mass relation). Then, $\bar{\beta}_{\tilde{\tau}}$ is found to be $\sim 0.93$. Thus, a relatively larger uncertainty of $\Delta \bar{\beta}_{\tilde{\tau}} \sim 0.05$ is expected if the dominant SUSY process cannot be understood.

To see how this affects the determination of the lifetime, we calculate the $\chi^{2}$ variable using the template with $\bar{\beta}_{\tilde{\tau}}=0.83$ and 0.93 . The results are shown in Figs. 5 and 6. We can see that error related to the uncertainty to the averaged velocity is $\sim 50-100 \mu \mathrm{m}$ if $\Delta \bar{\beta}_{\tilde{\tau}} \sim 0.05$. Even with such an uncertainty, we can still have a relatively good determination of $\tau_{\tilde{\tau}}$. Thus, the impact parameter will be a powerful tool to measure the lifetime at the LHC.

Finally, we consider how well we can estimate the gravitino mass if we assume that $\tilde{\tau}_{1}$ decays into the gravitino and $\tau$-lepton. In the case with the underlying gravitino mass of $9.7 \mathrm{eV}$, we have shown that the lifetime of the stau is estimated between $410 \mu \mathrm{m}$ and $540 \mu \mathrm{m}$ for $\bar{\beta}_{\tilde{\tau}}=0.88$. In addition, the uncertainty related to $\bar{\beta}_{\tilde{\tau}}$ is estimated to be about $40 \mu \mathrm{m}$, as one can see from Figs. 5 and 6. Then, by using the measured stau mass $m_{\tilde{\tau}}=120.5 \pm 18.1 \mathrm{GeV}$ and the center value of the estimated NLSP lifetime $c \tau_{\tilde{\tau}}=475 \mu \mathrm{m}$, we obtain $m_{3 / 2}=8.3 \pm 1.8 \pm 0.8 \pm 1.0 \mathrm{eV}$, where the errors originate in the uncertainties of $m_{\tilde{\tau}}, c \tau_{\tilde{\tau}}$ and $\bar{\beta}_{\tilde{\tau}}$, respectively. 


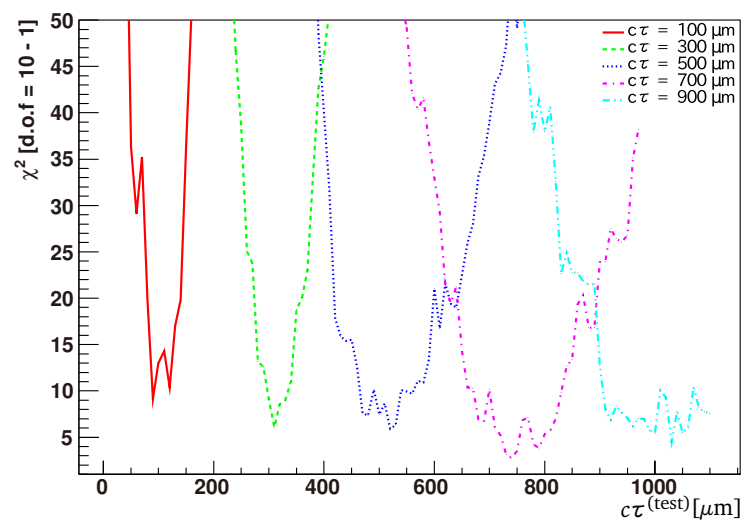

Figure 5: Same as Fig. 4, except for $\bar{\beta}_{\tilde{\tau}}=0.83$.

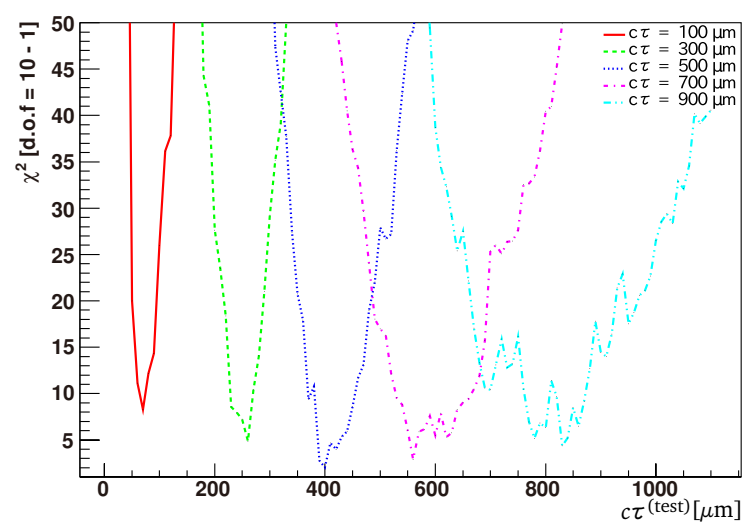

Figure 6: Same as Fig. 4, except for $\bar{\beta}_{\tilde{\tau}}=0.93$.

\section{Summary}

We have proposed a method to determine the mass spectrum of sparticles and the lifetime of the stau NLSP at the LHC when the mass of the gravitino LSP is of the order of $10 \mathrm{eV}$. Though the decay length of the stau NLSP is very short, which is of the order of $100-1000 \mu \mathrm{m}$, it is still possible to deeply study the model by utilizing the transverse impact parameter of tau-jets from the decay of the stau NLSP.

We have first discussed the mass measurement of sparticles using a typical cascade decay chain of a squark shown in Fig. 1. This SUSY event involves, at least, four $\tau$-leptons, which makes it difficult to analyze the signal because of combinato- 
rial backgrounds. Information about the impact parameter of the tau-jet, however, resolves the problem, and we have shown that the mass spectrum of sparticles can be determined accurately through the kinematical endpoints of $M_{\tau \tau}, M_{j \tau}$ (near), and $M_{T 2, j j}$.

We have also discussed the determination of the lifetime of the stau NLSP using the distribution of the transverse impact parameter. The impact parameter depends not only on the lifetime but also on the velocity of the stau NLSP. We have therefore developed a strategy to estimate the velocity by utilizing a simulation with information about the mass spectrum obtained in the previous stage. We have shown that, if the velocity distribution of $\tilde{\tau}_{1}$ is somehow understood, the lifetime of the stau NLSP is determined with the accuracy of about $30 \%$ as far as its decay length is larger than $\sim 100 \mu \mathrm{m}$. Thus, if the underlying model of the SUSY breaking is low-scale gauge mediation with the gravitino mass of $\mathcal{O}(10) \mathrm{eV}$, the LHC may have a chance to acquire some information about the gravitino mass.

\section{Acknowledgments}

This work is supported by Grant-in-Aid for Scientific research from the Ministry of Education, Science, Sports, and Culture (MEXT), Japan, Nos. 21740174 \& 22244031 (S.M.), No. 22540263 (T.M.), and No. 22244021 (M.A., S.M. and T.M.), by World Premier International Research Center Initiative (WPI Initiative), MEXT, Japan, and by JSPS Research Fellowships for Young Scientists, MEXT, Japan (T.I.). M.A. also acknowledges support from the German Research Foundation (DFG) through grant BR 3954/1-1

\section{References}

[1] See, for example, M. Drees, R. M. Godbole and P. Roy, Theory and phenomenology of sparticles, (World Scientific, 2004).

[2] M. Dine, A. E. Nelson, Phys. Rev. D48, 1277 (1993), M. Dine, A. E. Nelson, Y. Shirman, Phys. Rev. D 51, 1362 (1995), M. Dine, A. E. Nelson, Y. Nir, Y. Shirman, Phys. Rev. D 53, 2658 (1996).

[3] J. L. Feng, M. Kamionkowski and S. K. Lee, Phys. Rev. D 82, 015012 (2010). 
[4] M. Kawasaki, K. Kohri, T. Moroi and A. Yotsuyanagi, Phys. Rev. D 78 (2008) 065011.

[5] M. Viel, J. Lesgourgues, M. G. Haehnelt, S. Matarrese, A. Riotto, Phys. Rev. D71, 063534 (2005).

[6] S. Matsumoto and T. Moroi, Phys. Lett. B 701 (2011) 422.

[7] G. Aad et al. [The ATLAS Collaboration], arXiv:0901.0512 [hep-ex].

[8] G. L. Bayatian et al. [CMS Collaboration], CMS-TDR-008-1 (2006).

[9] K. Ishiwata, T. Ito and T. Moroi, Phys. Lett. B 669, 28 (2008).

[10] S. Kaneko, J. Sato, T. Shimomura, O. Vives and M. Yamanaka, Phys. Rev. D 78, 116013 (2008).

[11] S. Asai, T. Moroi and T. T. Yanagida, Phys. Lett. B 664, 185 (2008) arXiv:0802.3725 [hep-ph]].

[12] S. Asai, Y. Azuma, M. Endo, K. Hamaguchi and S. Iwamoto, arXiv:1103.1881 [hep-ph].

[13] See, Y. Kats, P. Meade, M. Reece, D. Shih, arXiv:1110.6444 [hep-ph]] and references therein.

[14] See, for example, I. Hinchliffe, F. E. Paige, M. D. Shapiro, J. Soderqvist, W. Yao, Phys. Rev. D55, 5520-5540 (1997).

[15] I. Hinchliffe, F. E. Paige, Phys. Rev. D60, 095002 (1999).

[16] C. G. Lester and D. J. Summers, Phys. Lett. B 463 (1999) 99.

[17] F. E. Paige, S. D. Protopopescu, H. Baer and X. Tata, arXiv:hep-ph/0312045.

[18] G. Corcella et al., JHEP 0101, 010 (2001); G. Corcella et al., arXiv:hep-ph/0210213.

[19] S. Moretti, K. Odagiri, P. Richardson, M. H. Seymour and B. R. Webber, JHEP 0204, 028 (2002).

[20] For information on Pretty Good Simulation of high energy collisions (PGS4), see http://www. physics . ucdavis . edu/\%7Econway/research/research.html.

[21] S. Jadach, J. H. Kuhn, Z. Was, Comput. Phys. Commun. 64, 275-299 (1990), N. Davidson, G. Nanava, T. Przedzinski, E. Richter-Was, Z. Was, arXiv:1002.0543 [hep-ph]]. 